\title{
A CHARACTERIZATION OF ClOSED MAPS USING THE WHYBURN CONSTRUCTION
}

\author{
YVONNE O. STALLINGS \\ Department of Mathematics \\ University of Southwestern Louisiana \\ Lafayette, Louisiana 70504
}

(Received May 22, 1984)

\begin{abstract}
In this paper we modify the Whyburn construction for a continuous function $f: X \rightarrow Y$. If the range is first countable, we get a characterization of closed maps; namely, the constructions are the same if and only if the map is closed.
\end{abstract}

KEY WORDS AND PHRASES. clused maps, first countable, Whyburn construction. 1980 MATHEMATICS SUBJECT CLASSIFICATION CODE. Primary 54A10, 54C10.

\section{INTRODUCTION.}

Let $f: X \rightarrow Y$ be continuous and let $X$ and $Y$ be Hausdorff. In [3] Whyburn defined the unified space $Z$ to be the disjoint union of $X$ and $Y$ with a set $Q$ open in $Z$ if and only if $Q \cap X$ is open in $X, Q \cap Y$ is open in $Y$, and for any compact $K \subset Q \cap Y, f^{-1}(K)-Q$ is compact. In this paper we modify the topology on $\mathrm{X} U \mathrm{Y}$ by defining $\mathrm{Q}$ to be open if and only if $Q \cap \mathrm{X}$ is open in $\mathrm{X}, Q \cap Y$ is open in $Y$, and for any point $p \varepsilon Q \cap Y, f^{-1}(p)-Q$ is compact. We denote the modified Whyburn space by $W$.

It is obvious that any set open in $Z$ is open in $W$. We will show that if $f$ is closed, the topologies are in fact the same and if $Y$ is first countable, then $Z$ and $\mathrm{W}$ being the same implies that $f$ is closed. This will yield the following corollary:

COROLLARY. Any continuous function from a Hausdorff space into the reals (or any metric space) is closed if and only if the Whyburn space and the modified Whyburn space are the same.

\section{PRELIMINARIES.}

Arguments similar to those of Whyburn's show that $W$ is a $T_{1}$ topological space containing $\mathrm{X}$ as an open subspace and $\mathrm{Y}$ as a closed subspace. However, 
just as in the Whyburn space, W need not be Hausdorff. Whyburn showed that $Z$ is Hausdorff if $X$ is locally compact. Asking when $W$ is Hausdorff led to the following definitions and propositions:

DEFINITION 2.1. Let $f: X \rightarrow Y$ be continuous. Then $A \subset X$ is fiber compact if and only if $A$ is closed and for all y $\varepsilon f(A), f^{-1}(y) \cap A$ is compact. Also, $X$ is locally fiber compact if every point has a neighborhood whose closure is fiber compact.

PROPOSITION 2.2. If $A$ is fiber compact in $X$, then $W-A$ is open. PROOF. Since $A$ is closed in $X,(W-A) \cap X$ is open in $X$; also $(\mathrm{W}-\mathrm{A}) \cap \mathrm{Y}=\mathrm{Y}$ is open in $\mathrm{Y}$. Now let $\mathrm{p}$ be any point in $\mathrm{Y}$. Then $f^{-1}(p)-(W-A)=f^{-1}(p) \cap A$ which is compact since $A$ is fiber compact.

PROPOSITION 2.3. If $X$ is locally fiber compact, then $W$ is Hausdorff. PROOF. The only interesting case is when $p$ is in $X$ and $f(p)=q$. Since $X$ is locally fiber compact, there exists a $U$ open in $X$ such that $p$ is in $U$ and $\bar{U}$ is fiber compact. Hence $U$ is open in $W$ and $W-\bar{U}$ is a neighborhood of $q$ by Proposition 2.2.

We define, as did Whyburn, a retraction $\mathrm{r}: \mathrm{W} \rightarrow \mathrm{Y}$ to be $\mathrm{f}$ on $\mathrm{X}$ and the identity on $Y$. The following results parallel those of Whyburn's for $r: Z \rightarrow Y$. The proof is omitted.

PROPOSITION 2.4. The map $\mathrm{r}: \mathrm{W} \rightarrow \mathrm{Y}$ is continuous, has compact fibers and is closed (open) if $f$ is.

The next proposition shows that some of the properties mentioned above actually characterize the modified Whyburn construction. This proposition is similar to a theorem about the Whyburn construction proved by Dickman [1].

PROPOSITION 2.5. Let $\mathrm{r}: \mathrm{S} \rightarrow \mathrm{Y}$ be a retraction with compact fibers from a Hausdorff space onto a regular subspace. Let $X=S-Y$ and $f=r \mid X^{*}$ If fiber compact subsets of $X$ are closed in $S$, then the modified Whyburn space for $f: X \rightarrow Y$ is homeomorphic to $S$.

PROOF. Let $W$ be the modified Whyburn space for $f: X \rightarrow Y$. If $V$ is open in $S$ and $p$ is any point in $V \cap Y$, then $r^{-1}(p)$ is compact. But $r^{-1}(p)-V$ $=f^{-1}(p)-V$ and therefore $V$ is open in $W$.

Now let $\mathrm{Q}$ be open in $\mathrm{W}$ and let $\mathrm{x} \varepsilon \mathrm{Q}$. If $\mathrm{x} \varepsilon \mathrm{X}$, then $\mathrm{Q} \cap \mathrm{X}$ is an open set in $S$ and is contained in $Q$. Suppose $x \varepsilon Q \cap Y$. Then since $Y$ is regular, we can find a neighborhood $V$ of $x$ such that $x \varepsilon V \subset \bar{V} \subset Q \cap Y$. Let $f^{-1}(\bar{V})-Q=B$. Then $B$ is fiber compact and so $S-B$ is open in $S$. Let $U=(S-B) \cap r^{-1}(V)$. Then $U$ contains $x$, is open in $S$, and is contained in $Q$. 3. MAIN THEOREM.

We now state and prove the major theorem of this paper which allows us to determine when $W$ and $Z$ are the same. 
THEOREM 3.1. Let $f: X \rightarrow Y$ be continuous, $X$ and $Y$ be Hausdorff, and let $Y$ be first countable. Then $Z$ and $W$ are equal if and only if $f$ is a closed mapping.

PROOF. Assume $f$ is closed, $Q$ is open in $W$ and $K$ is any compact subset of $Q \cap Y$. Let $f^{-1}(K)-Q=A$. Then $A$ is closed and $f(A)$ is a closed subset of $K$ and hence is compact. Then $f_{\mid A}: A \rightarrow f(A)$ is a continuous, closed surjection with compact fibers and therefore is a perfect map. By [2, Theorem 5.3] $A$ is compact. Hence $Q$ is open in $Z$.

Now assume that $\mathrm{Z}$ and $\mathrm{W}$ are equal. Let $\mathrm{A}$ be a closed set in $\mathrm{X}$. Suppose that $y$ is a limit point of $f(A)$. Since $Y$ is first countable and Hausdorff, there exists a sequence of distinct points $\left\{y_{n}\right\} \subset f(A)$ which converges to y. So we may choose a sequence $\left\{x_{n}\right\}$ in $A$ such that $f\left(x_{n}\right)=y_{n}$.

Let $B=\left\{x_{n}\right\}$. Now suppose $B$ has no limit points. Then $B$ is closed in $X$. Since for any $y_{n} \varepsilon f(B), f^{-1}\left(y_{n}\right) \cap B=\left\{x_{n}\right\}, B$ is fiber compact and thus $W-B$ is open in $W$ by Proposition 2.2. Since $Z=W, Z-B$ is open in $Z$. Now $K=\left\{y_{n}\right\} U\{y\}$ is a compact subset of $Y \cap(Z-B)$; therefore, $\mathrm{f}^{-1}(\mathrm{~K})-(Z-\mathrm{B})=\mathrm{B}$ is compact. Since $\mathrm{B}$ is also infinite it must have a limit point, contradicting our assumption. Hence $B$ has a limit point, say $x$.

Suppose that $f(x)=z \neq y$. Then we can find disjoint neighborhoods $V$ of $z$ and $U$ of $y$. Since $\left\{y_{n}\right\}$ converges to $y$, there exists an $N$ such that for every $\mathrm{n} \geq \mathrm{N}, \mathrm{y}_{\mathrm{n}} \varepsilon \mathrm{U}$. However, since $\mathrm{x}$ is a limit point of $\mathrm{B}$, we have an integer $\mathrm{m}>\mathrm{N}$ such that $\mathrm{x}_{\mathrm{m}} \varepsilon \mathrm{f}^{-1}(\mathrm{~V})$. Hence $f\left(\mathrm{x}_{\mathrm{m}}\right)=\mathrm{y}_{\mathrm{m}}$ is in both $U$ and $\mathrm{V}$ which is impossible. Hence $f(x)=y$. Since $B \subset A$ and $A$ is closed, $x \varepsilon A$ and there.. fore $f(A)$ is closed.

Notice that $Y$ being first countable is a necessary hypothesis for the preceding theorem. The following is an exam ple to illustrate this.

Let $X_{i}=[0,1)$ for all $i=1,2,3, \ldots$. Then let $X$ be the disjoint union of these $X_{i}^{\prime} s$. Let $Y=X U p$ where $p$ is not in $X$. Define $V \subset Y$ to be open if and only if

1) $\mathrm{V}$ is an open set contained in $\mathrm{X}$ or

2) If $p \varepsilon V$, then there exists a finite set of indices such that if $i \varepsilon\left\{i_{1}, \ldots, i_{n}\right\}$ then $x_{i}-V=x_{i}$ and if $i \varepsilon\left\{i_{1}, \ldots, i_{n}\right\}$ then $x_{i}-V$ is compact. The inclusion map from $X$ to $Y$ is not closed, $Y$ is not first countable at $\mathrm{p}$ and yet $\mathrm{W}$ and $\mathrm{Z}$ are the same.

\section{REFERENCES}

1. DICKMAN, R. F., JR., Unified Spaces and Singular Sets for Mappings of Locally Compact Spaces, Fundamenta Mathematicae, 62 (1968), 103-123.

2. DUGUNDJI, J., Topology. Boston, Allyn and Bacon (1966).

3. WhyBURN, G.T., A Unified Space for Mappings, Trans. Amer. Math. Soc. $\underline{74}(1953), 344-350$. 


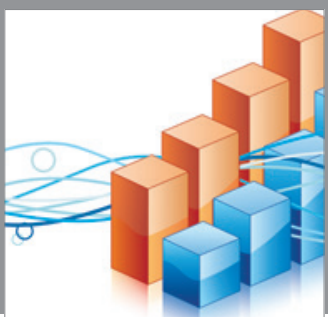

Advances in

Operations Research

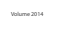

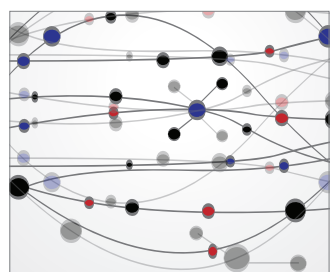

\section{The Scientific} World Journal
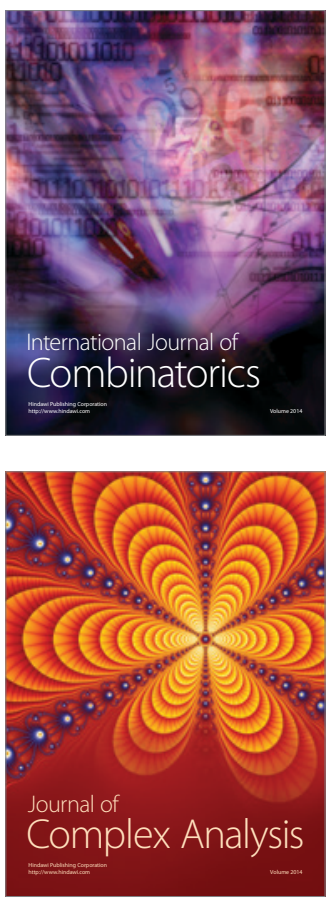

International Journal of

Mathematics and

Mathematical

Sciences
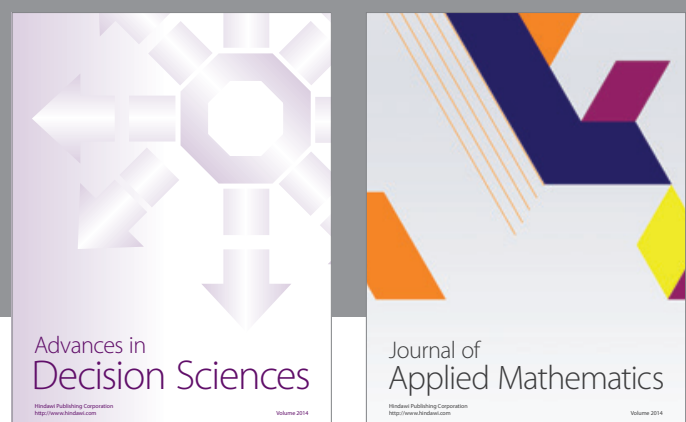

Journal of

Applied Mathematics
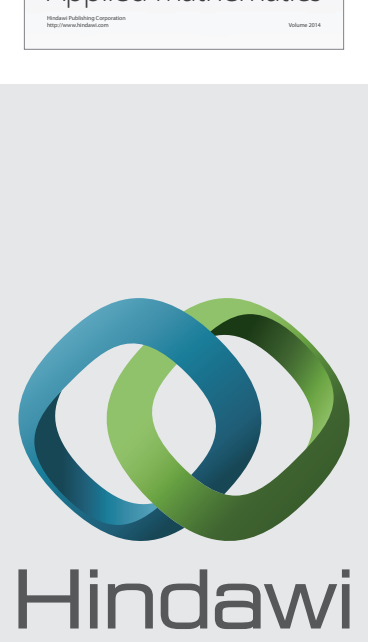

Submit your manuscripts at http://www.hindawi.com
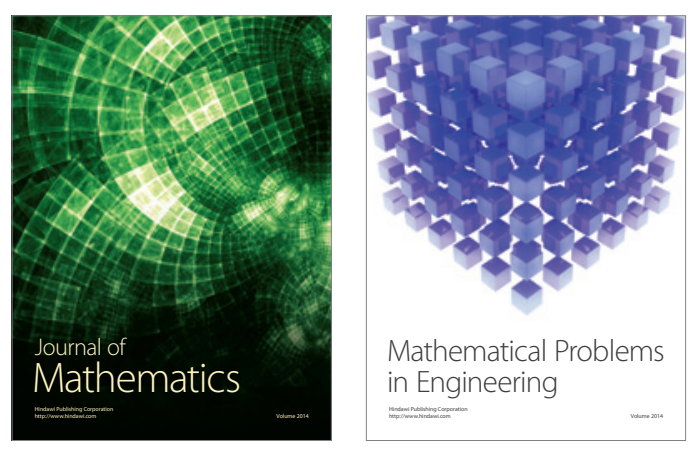

Mathematical Problems in Engineering
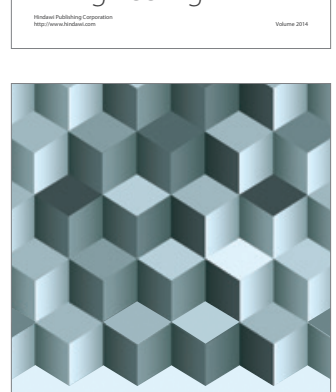

Journal of

Function Spaces
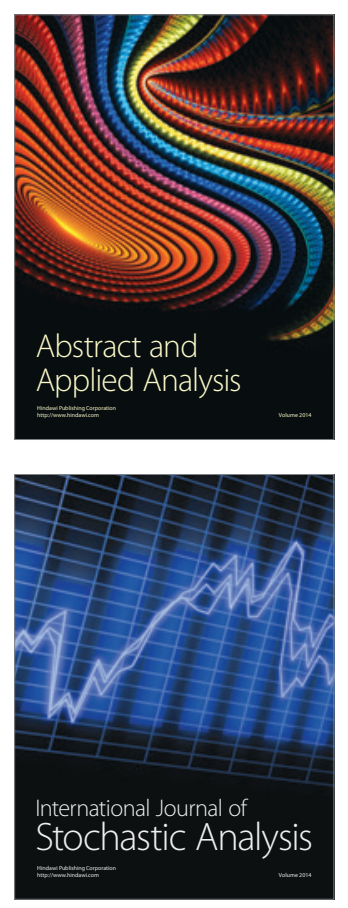

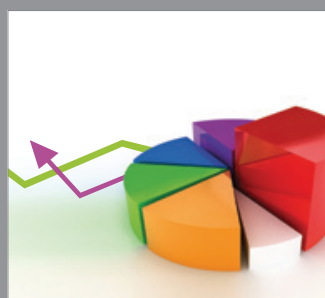

ournal of

Probability and Statistics

Promensencen
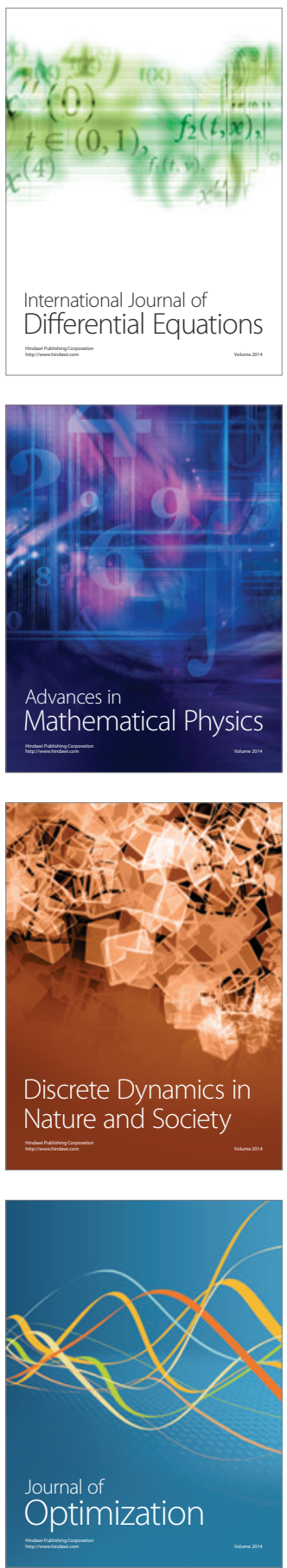